\title{
CONTEXTO COMUNICATIVO, FAMILIAR Y ESCOLAR DEL ADOLESCENTE Y CONDUCTA SEXUAL.
}

\author{
Serafín J. Cruces Montes. \\ Rocío Guil Bozal. \\ Sergio Sánchez Sevilla. \\ Antonio Zayas García. \\ Universidad de Cádiz. \\ serafin.cruces@uca.es
}

https://doi.org/10.17060/ijodaep.2017.n1.v2.916

Fecha de Recepción: 20 Enero 2017

Fecha de Admisión: 1 Abril 2017

\section{RESUMEN}

Family and school adolescent communicative context, and sexual behaviour.

La adolescencia es considerada como un periodo especialmente sensible en el proceso evolutivo del ser humano. Es en este periodo donde se van a adquirir determinados comportamientos denominados de riesgo para el adolescente. El objetivo fundamental se centra en analizar el contexto comunicativo, familiar y escolar en relación al comportamiento sexual del adolescente (debut, uso de medidas anticonceptivas y conocimiento de enfermedades sexuales). Se les administró a alumnos de E.S.O. (Enseñanza Secundaria Obligatoria) de toda Andalucía un cuestionario en donde se abordaban factores demográficos, familiares y educativos; así como diversas cuestiones relacionadas con su conducta sexual. Los resultados muestran que de manera mayoritaria, los adolescentes han recibido educación sexual, valorada muy positivamente, pero que sin embargo no resulta determinante para el uso de métodos anticonceptivos aunque si facilita el conocimiento de enfermedades de transmisión sexual. Aquellos que han recibido educación sexual se inician antes en prácticas sexuales de riesgo. El preservativo es el método más utilizado siendo el motivo de uso evitar un embarazo no deseado. Los que se inician precozmente en las relaciones sexuales poseen padres con menor nivel de estudios, manifiestan una peor relación con los mismos tanto a nivel de comunicación, como de control y disciplina y poseen peores resultados académicos. Los resultados obtenidos muestran la importancia de los factores familiares y educativos a la hora de iniciarse los adolescentes en su vida sexual de una manera temprana.

Palabras clave.

Adolescencia, Sexualidad, Factores Protectores, Familia, Padres, Profesorado, Comunicación y Educación. 


\section{ANTECEDENTES}

La adolescencia se presenta como una etapa del desarrollo donde el sujeto adquiere comportamientos y estilos de vida saludables (Newman, Harrison, Dashiff y Davies, 2008). Es en este periodo donde se producen cambios de toda índole en el adolescente, apareciendo además un conjunto de conductas de riesgo, entre las que se encuentra la iniciación en comportamientos sexuales. Los jóvenes comienzan su actividad sexual por la presión de compañeros, la necesidad de comunicar afecto, el evitar encontrarse en soledad, el buscar autoestima y afecto, el hecho de ser valorados, el demostrar su nivel de independencia y el ir en contra de la autoridad, en definitiva demostrar a los padres que ya se es adulto (Temple-Smith, Moore, y Rosenthal, 2015).

Ante el hecho de reconocer que los jóvenes tienen su primera relación como inesperada hace difícil la adopción de medidas protectoras que hagan de la relación sexual una conducta saludable (Espada, Quiles y Méndez, 2003). La existencia de embarazos no deseados, el hecho de que las mujeres jóvenes de entre 15-24 años se hallen en una situación de mayor riesgo de infección del VIH (UNAIDS, 2016), el aumento de otro tipo de infecciones (Infección gonocócica y sífilis) (Instituto de Salud Carlos III, 2011) y el hecho de que las jóvenes, al no tener su cervix madurada, son más vulnerables a las ETS vinculadas a la enfermedad inflamatoria pélvica y susceptibles de infección del Virus del Papiloma Humano, muestra la necesidad de tener en cuenta el uso de métodos de protección, la ocurrencia de múltiples parejas y la realización de prácticas de riesgo a fin de poder evitar los riesgos planteados (Espada, Escribano, Orgilés, Morales, \& Guillén-Riquelme, 2015; Teva, Bermúdez Buela-Casal 2009)

Parece, por tanto, importante tener presente la existencia de determinadas variables y contextos como pueden ser el estatus socioeconómico de los padres, la edad, el tipo de hábitat y el centro educativo tal y como se refleja en el trabajo HBSC (Health Behaviour in School-aged Children) (Moreno et al. 2011) ya que resulta importante determinar la influencia de los factores familiares, escolares y económicos que afectan a al debut sexual y a la realización de prácticas sexuales de riesgo (Erkut et al., 2013).

En esta línea Manlove, Ryan y Franzetta (2007) destacan la existencia de factores individuales y escolares que determinan la adaptación de los adolescentes a la sociedad en la que se desenvuelven y que influyen en su debut sexual. El nivel educativo correlaciona inversamente con el inicio temprano en el sexo; también se relaciona con un inicio temprano el tener bajas calificaciones y por expulsiones continuas del colegio. Sabia (2007) señala que los adolescentes que han mantenido relaciones sexuales presentan menos apego al colegio que el resto. A mayor adaptación escolar, el inicio sexual es más tardío (Castro, Bermúdez, Buela-Casal y Madrid, 2011). Los adolescentes que están bien integrados escolarmente, es decir que participan académicamente, conectan con los iguales y profesores, y participan en actividades curriculares, tienen menos riesgo de actividad sexual temprana, retrasando dicha actividad sexual (Kotchick, Shaffer, Forehand y Miller, 2001). Por el contrario una baja autoestima, un mal ajuste psicosocial, y un pobre desarrollo académico influyen en el inicio sexual temprano (Silver y Bauman, 2006). El contexto escolar, por tanto, constituye uno de los principales agentes de socialización, así como fuente institucionalizada de educación sexual para el adolescente.

No menos importancia tiene el medio familiar en relación con el inicio de la conducta sexual en la adolescencia. La familia, su estructura, disciplina y niveles de comunicación parecen tener un papel determinante en la realización de conductas sexuales de riesgo en la adolescencia (Andrade, Betancourt y Palacios, 2006; Lameiras, Rodríguez, Calado y González, 2004; Palacios y Andrade, 2006; Lindberg, Maddow-Zimet, y Boonstra, 2016; Cueto \& Leon, 2016). Algunos autores señalan que niños cuya infancia ha tenido una historia de conductas agresivas y disruptivas (inatención e 
hiperactividad) está asociado con la temprana iniciación de relaciones y elevadas puntuaciones de actividad sexual adolescente entre chicos (Barkley, Fischer, Smallish y Fletcher, 2006) y temprana iniciación de relaciones, múltiples parejas sexuales e incrementos de embarazos no deseados (Ramrakha et al., 2007).

DiClemente et al. (2008) señalan que la conducta de los adolescentes se encuentra asociada a la estructura familiar. La familia y más concretamente el afecto y cuidado de los padres promociona la salud, empoderamiento y felicidad, influyendo en las conductas sexuales de riesgo de sus hijos y retrasando la aparición de relaciones sexuales en los adolescentes (Ferreira et al., 2013).

El apoyo de la familia, su cohesión, la supervisión parental, y la comunicación entre padres y adolescentes acerca de sexo son elementos que ayudan a prevenir a los adolescentes en la participación de conductas sexuales de riesgo. La supervisión parental resulta muy importante ya que los jóvenes que perciben que sus padres (o la figura paterna/materna) saben donde se encuentran y con quienes están fuera de casa, son menos probables a participar en conductas de riesgo de ETS/SIDA (Crosby, DiClemente, Wingood, Lang, y Harrington 2003). Cavazos-Rehg, et al. (2010) ponen de manifiesto que el vacío de supervisión y apoyo de los padres, la limitada conexión familiar, la discordia marital o de la pareja y la pobre calidad de las relaciones padres-hijos, todas éstas han sido correlacionadas con resultados negativos conductuales, incluyendo la temprana actividad sexual entre niños y adolescentes. Estos autores señalan que tanto los factores del sistema (género, étnia, estatus socioeconómico, entornos urbanos, estatus durante la infancia, dependencia de alcohol y conducta desordenada) y los factores familiares (ingesta de alcohol en casa, background religioso, background educacional de la madre y el padre, y ser hijo de una chica adolescente) afectan al precoz debut sexual.

Por último, señalar al grupo de iguales como otro factor importante de influencia en la adolescencia (DiClemente, et al. 2008). Si los adolescentes y jóvenes adultos perciben que su grupo de iguales están teniendo sexo sin protección o participando en conductas de riesgo sexual, éstos serán más probables que adopten los comportamientos de su grupo de iguales. El hecho de que los compañeros sean activos sexualmente hace que los chicos y chicas ignoren los mensajes de abstinencia (Silver y Bauman, 2006).

\section{OBJETIVOS DE LA INVESTIGACIÓN.}

El presente trabajo tiene como objetivo principal conocer una descripción de la conducta sexual de los adolescentes andaluces estudiantes de ESO (Enseñanza Secundaria Obligatoria) en relación a determinadas variables sociodemográficas, comunicativas, académicas y familiares.

Como objetivos secundarios de nuestro trabajo planteamos conocer el conjunto de variables en relación al comportamiento sexual referido a los jóvenes andaluces estudiantes de ESO; conocer si han recibido educación sexual, su valoración y por parte de quién o quiénes; conocer el número de parejas y el método utilizado preferentemente por los jóvenes andaluces estudiantes de ESO en su relaciones sexuales; y por último saber el conocimiento que tienen de las enfermedades de transmisión sexual.

\section{MUESTRA Y/O PARTICIPANTES.}

La población objeto de estudio fue la población de estudiantes de ESO de la Comunidad Autónoma Andaluza. El total de la muestra del presente trabajo está constituida por un total de 2225 sujetos estudiantes de ESO de la Comunidad Autónoma Andaluza con una media de edad de 14.33 años y una DT de 1.38 La muestra se distribuyó de la siguiente manera. Un 50,8\% de chicos y $49.2 \%$ de chicas; los valores de edad se distribuyeron de la siguiente manera: con 12 años un 
10.7\%; con 13 años un 19.7\%; con 14 años un 22.6\%; con 15 años un $25.5 \%$; con 16 años un 16.9\%; con 17 años un 4\% y con 18 años un 0.6\%. Media de edad 14.3; edad máxima 18 años y mínima de 12 años. La muestra fue obtenida a través de un diseño muestral probabilístico con estratificación realizada en dos fases: la primera según la población estudiantil de ESO en relación proporcional a la población y tipos de Centros e Institutos de Enseñanza Media de la Comunidad; en la segunda fase se realizó la distribución proporcional según los grupos de sexo y edad.

\section{METODOLOGÍA Y/O INSTRUMENTOS UTILIZADOS.}

Al fin de obtener los datos necesarios para la cobertura de nuestros objetivos con el presente trabajo, se administró en los distintos centros de secundaria un cuestionario ex profeso, en los cuatro niveles de ESO, de toda la comunidad autónoma, el cual se describe a continuación

El cuestionario comprendía 102 preguntas que aglutinaba 199 variables abarcando cuestiones demográficas, académicas, familiares y de ocio. Evalúa aspectos tales como, la composición de la familia, nivel formativo de los progenitores, percepción de la situación económica, valoración de los miembros de la unidad familiar y amigos, valoración sobre los valores, elementos de disciplina y control ejercido por los padres, valoración de los niveles de comunicación con los padres y madres, percepción sobre existencia de problemas en el seno familiar en relación a discusiones violentas entre los padres, padecimiento de malos tratos por parte de los padres y la existencia de problemas por el consumo de alcohol y drogas en casa.

En relación a los datos del contexto educativo se les preguntaba sobre la valoración que hacían de la sensibilidad hacia las opiniones y necesidades de los alumnos por parte de los profesores, así como el nivel de comprensión y comunicación sobre las ideas que estos les transmiten; el nivel de asistencia a clase así como las calificaciones y realización de actividades extraescolares.

El trabajo que presentamos formaba parte de un proyecto más global sobre el análisis de los distintos comportamientos de riesgo en la adolescencia cuyas primeras valoraciones fueron analizadas (Cruces, 2012; Rodríguez y Traverso, 2012; Rodríguez, et al. 2008). En nuestro caso indicaremos el apartado que recogía información sobre el comportamiento sexual de los adolescentes (edad de comienzo de relación sexual, orientación sexual, educación sexual recibida, tenencia de pareja, uso de métodos anticonceptivos, prácticas sexuales y conocimiento de ETS).

La validez del instrumento fue llevada a cabo por investigadores pertenecientes a grupos del Plan Andaluz de Investigación, que lo valoraron y determinaron la validez de su contenido (Salazar, Varela, Tovar y Cáceres, 2006). Así mismo el cuestionario se sometió a una prueba piloto. La confiabilidad (coeficiente de Cronbach) fue de 0.70 . Fueron validadas 2225 encuestas.

\section{RESULTADOS ALCANZADOS.}

En cuanto a las variables relacionadas con la conducta sexual del adolescente, comenzar señalando que un $41.3 \%$ dice tener pareja o ligue, frente al $53.8 \%$ que dice no tenerla. Siendo de manera significativa y mayoritaria las mujeres, con un $54.1 \%$ las que manifiestan tener pareja.

El $60.9 \%$ de los participantes manifiestan haber recibido educación sexual frente a un $32.9 \%$ que señala no haberla recibido, no habiendo diferencias significativas por sexo.

Si lo analizamos por curso de ESO encontramos una relación significativa, $\chi^{2}(3)=257.23$, $\mathrm{p}=0.00$ en donde podemos observar que de $1^{\circ}$ de ESO un 38.8\% dicen haber recibido educación sexual, en $2^{\circ}$ de ESO $61.1 \%$, de $3^{\circ}$ un $78.1 \%$ y de $4^{\circ}$ un $80.6 \%$.

La valoración que hacen de esa educación sexual es de 7.36 puntos, sobre 10, de media y 2.06 de DT. No mostrándose diferencias significativas entre sexos. Al realizar un ANOVA de un factor con comparaciones post hoc, no encontramos diferencias significativas entre los distintos cursos de 
ESO en la valoración que hacen los alumnos de la variable "Valoración de la educación sexual recibida". En este sentido, aquellos que han mantenido relaciones sexuales valoran mejor la educación sexual recibida que los que no la han mantenido, $t(1310)=3.12, p=0.00)$.

Cuando se les preguntó sobre el principal medio a través del cual reciben información sobre sexualidad y métodos anticonceptivos, las respuestas se distribuyen según la siguiente la figura 1.

Figura 1

\section{Fuentes información sexual}

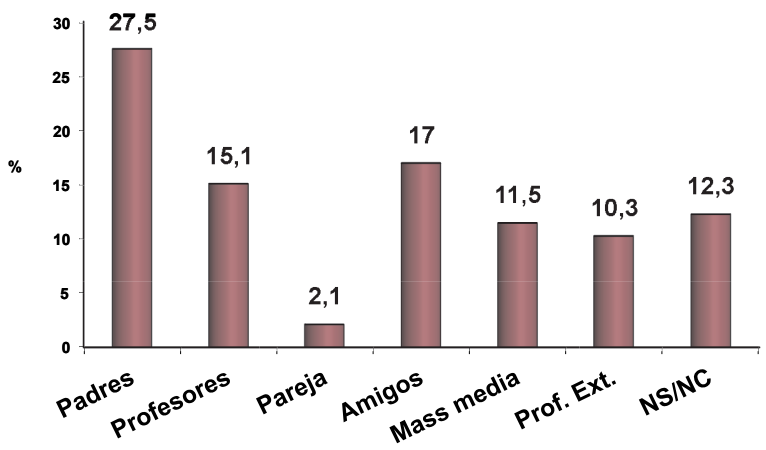

Podemos ver como los padres y luego los amigos (27.5\% y $17 \%$, respectivamente) son las principales fuentes de información sobre sexualidad y métodos anticonceptivos, no encontrándose diferencias significativas por sexos.

Las personas que dicen haber mantenido relaciones sexuales con penetración suponen el 17.4\% de los jóvenes de nuestra muestra. No se encuentran diferencias proporcionales significativas por sexo. La edad de inicio de la primera relación sexual es a los 14.28 años y 1.38 de DT, dándose diferencias significativas por sexo, donde los chicos tienen relaciones sexuales a una menor edad que las chicas (14.11 años vs. 14.48 años), $\mathrm{t}(344.69)=2.69, \mathrm{p}=0.00$.

Pasemos a ver la relación que se establece entre las distintas variables de contexto escolar, familiar y comunicativo y el haber mantenido relaciones sexuales completas.

Aquellos que han mantenido relaciones sexuales con penetración manifiestan que sus padres tienen un menor nivel de estudios que los que no han mantenido relaciones sexuales, $t$ (1743)=4.72, $p=0.00$. Los que han mantenido relaciones sexuales valoran peor la relación que mantienen con sus padres, y familia en general, $t(527,616)=-4.77, p=0.00$ y $t(1859)=-3.43, p=0.00$, respectivamente. De igual forma estos alumnos, conceden menos valor a los valores aportados por los padres, al igual que la disciplina/control y comunicación ejercida por ellos, $\mathrm{t}(513.925)=-3.21$, $\mathrm{p}=0.00 ; \mathrm{t}(2089)=-2.86, \mathrm{p}=0.00$ y $\mathrm{t}(2100)=-4.15, \mathrm{p}=0.00$ respectivamente. 
Tabla 1

Conducta sexual y variables familiares

\begin{tabular}{|c|c|c|c|c|c|}
\hline & $\begin{array}{l}\text { Hast terado } \\
\text { conto }\end{array}$ & $\mathrm{N}$ & Meda & $\begin{array}{c}\text { Derviación } \\
\text { be }\end{array}$ & $\begin{array}{l}\text { Erros tip de } \\
\text { la miedia }\end{array}$ \\
\hline \multirow{2}{*}{$\begin{array}{l}\text { Nivel cusisino } \\
\text { de eatubos pos } \\
\text { alguro de los } \\
\text { padtos }\end{array}$} & St & 332 & 2,64 & 927 & .051 \\
\hline & No & 1413 & 291 & .913 & .024 \\
\hline \multirow{2}{*}{$\begin{array}{l}\text { Valorwació } \\
\text { relación Gon } \\
\text { patres }\end{array}$} & Si & 380 & 8,02 & $1.87 \mathrm{t}$ & .096 \\
\hline & Do & 1730 & 8,52 & 1,714 & .041 \\
\hline \multirow{2}{*}{$\begin{array}{l}\text { Valor fexnalia } \\
\text { (pactres, } \\
\text { hemanosy } \\
\text { resto fandia) }\end{array}$} & Si & 340 & 7,9441 & 1,57844 & .08560 \\
\hline & $\mathrm{No}_{0}$ & 1521 & 8.2558 & 1,49945 & .03845 \\
\hline \multirow{2}{*}{$\begin{array}{l}\text { Valarsción de } \\
\text { Jou valozes gue } \\
\text { aportan los } \\
\text { padtes }\end{array}$} & St & 375 & 4,37 & .800 & .041 \\
\hline & $\mathrm{N}_{0}$ & 1715 & 4,51 & .318 & .013 \\
\hline \multirow{2}{*}{$\begin{array}{l}\text { Valoració de } \\
\text { la discidina y } \\
\text { control que } \\
\text { ejercen padtes }\end{array}$} & $\mathrm{Si}$ & 377 & 4.10 & .875 & .045 \\
\hline & No & 3714 & 4,23 & .805 & .019 \\
\hline \multirow{2}{*}{$\begin{array}{l}\text { Cominicacion } \\
\text { con postes }\end{array}$} & Si & 332 & 4,02 & .823 & 042 \\
\hline & No & 1730 & 4,20 & .797 & .019 \\
\hline
\end{tabular}

Tabla II

Contraste de medias conducta sexual y variables familiares

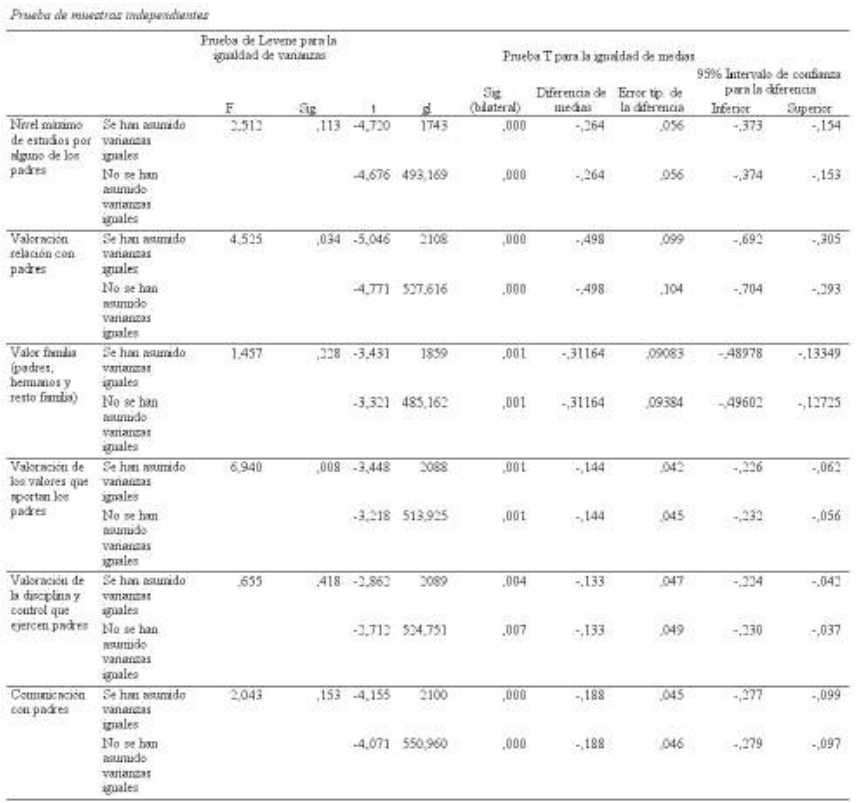

En relación con aspectos académicos, aquellos que mantienen relaciones sexuales sacan peores notas, perciben que los profesores no tienen en cuenta sus opiniones y comprenden peor las ideas que les son transmitidas por éstos, $t(574.962)=-9.42, p=0.00 ; t(554.018)=-7.91, p=0.00$ y $t(516.932)=-6.61, p=0.00$, respectivamente. 
Tabla III

Conducta sexual y variables académicas

Conducta sexual y variables académicas

\begin{tabular}{|c|c|c|c|c|c|}
\hline & $\begin{array}{l}\text { Has temido } \\
\text { conte }\end{array}$ & $\mathrm{N}$ & 1Meda & $\begin{array}{c}\text { Destiación } \\
\text { ty }\end{array}$ & $\begin{array}{l}\text { Frsor tip de } \\
\text { la medas }\end{array}$ \\
\hline \multirow{2}{*}{ Notas } & $S i$ & 388 & 285 & 1,142 & 058 \\
\hline & No & 1728 & 3.46 & 1,145 & 028 \\
\hline \multirow{2}{*}{$\begin{array}{l}\text { Frofesorea } \\
\text { tienen en cuenta } \\
\text { opinionca y } \\
\text { necesidades }\end{array}$} & $S \mathrm{i}$ & 380 & 2,98 & 1,000 & .051 \\
\hline & No & 1676 & 3.43 & .974 & .024 \\
\hline \multirow{2}{*}{$\begin{array}{l}\text { Comprendes } \\
\text { ideas } \\
\text { profesores }\end{array}$} & $\mathbf{S i}$ & 379 & 3.41 & .934 & .048 \\
\hline & No & 1718 & 3,76 & 828 & 020 \\
\hline
\end{tabular}

Tabla IV

Contraste de medias conducta sexual y variables académicas

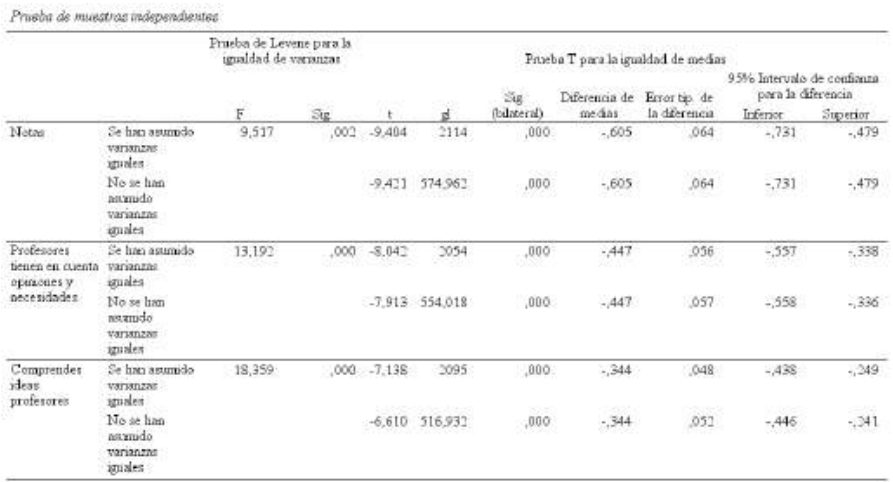

Siguiendo con las prácticas sexuales abordamos dos que pueden ser de riesgo potencial en relación a la transmisión de ETS o VIH/SIDA. La primera de ellas es la fellatio. En relación a esta práctica un $9 \%$ de los encuestados dice haberla practicado, siendo la edad promedio de inicio en la misma los 14.21 años. Vuelven los chicos a ser más precoces que las chicas en esta práctica (13.88 años vs. 14.65 años), $t(184.224)=-4.06, p=0.00)$. La otra práctica sexual de riesgo en la adolescencia sería el cunnilingus. El 8.6\% dice haber practicado dicha actividad sexual, siendo la edad de inicio de la misma de 14.30 años, no encontrándose diferencias estadísticamente significativas en función del sexo o género.

En relación a la primera vez que realizaron relaciones sexuales completas, el $13.9 \%$ señala que lo hizo con la pareja, $\chi^{2}(2)=392.29, p=0.00$ y un $2.6 \%$ con un amigo/a.

La distribución por sexos indica que los hombres en un 70\% señala que la primera vez lo hizo con su pareja y un $23.2 \%$ con un amigo/a; las mujeres en un $90.8 \%$ refieren que la primera vez lo hicieron con su pareja y un $7.2 \%$ manifiestan haberlo hecho con un amigo/a, $\chi^{2}(2)=26.46, p=0.00$.

El $14.7 \%$ de la muestra señala haber utilizado algún tipo de anticonceptivo en su primera relación sexual. Cabría esperar que aquellos que han recibido educación sexual utilizaran en mayor medida algún método anticonceptivo en su primera relación sexual. No obstante detectamos que tanto los que reciben educación sexual como los que no, manifiestan mayoritariamente hacer uso de un método anticonceptivo en su primera relación sexual. Para profundizar en este hallazgo sería 
interesante comprobar la valoración que sobre la educación sexual recibida realizan los que utilizan un método anticonceptivo en su primera relación sexual respecto a los que no. Hallamos que no hay diferencias significativas en la valoración sobre la educación sexual recibida y la utilización de métodos anticonceptivos en esa primera relación, $\mathrm{t}(294)=0.45, \mathrm{p}=0.65$.

En esta misma línea, obtenemos los mismos resultados entre aquellos que utilizan métodos anticonceptivos en la actualidad $t(283)=0.31, p=0.75$. De estos resultados podemos inferir que el recibir o no educación sexual no incide en el uso de medidas contraceptivas ni en la primera relación sexual ni en las siguientes.

En relación con la educación sexual, aquellos que dicen no haberla recibido han sido más precoces a la hora de tener relaciones completas, $t(363)=-3.82, p=0.00$, así como en las prácticas sexuales como la fellatio 0 el cunnilingus, $\mathrm{t}(184)=-2.71, \mathrm{p}=0.00$ y $\mathrm{t}(62.278)=-2.33, \mathrm{p}=0.02$, respectivamente.

Sobre los métodos de protección utilizados en esa primera vez, de los que dicen haber utilizado alguno encontramos los siguientes datos, el $97.9 \%$ señala el preservativo, el $0.6 \%$ píldora anticonceptiva y el $1.5 \%$ el coito interrumpido.

Figura 2

\section{Razón uso anticonceptivo primer coito}

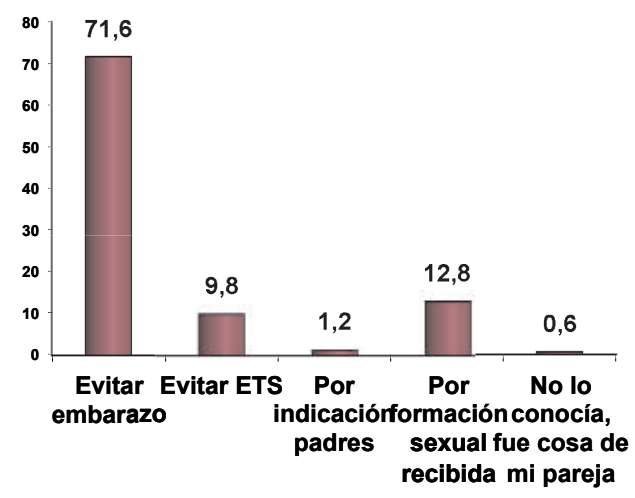

Figura 3

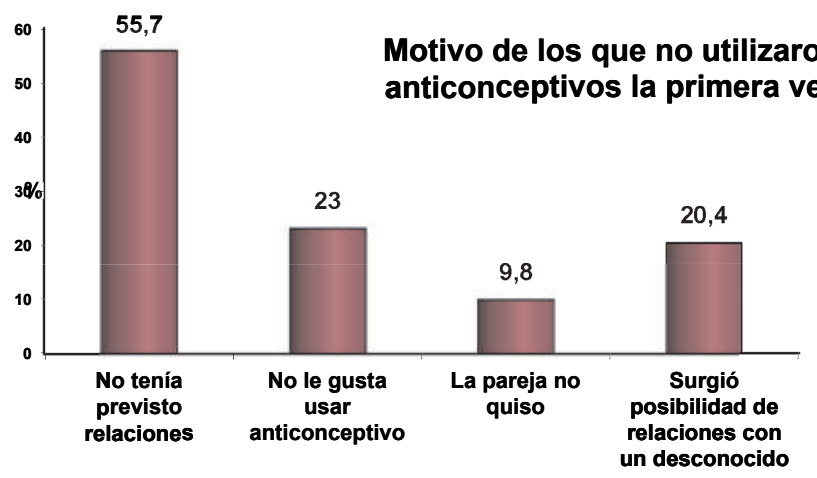


De los que dicen no haber usado métodos anticonceptivos cuando han mantenido una relación sexual, encontramos que la mayoría esgrime el no haber previsto tener relaciones.

En la actualidad el $11,8 \%$ de los encuestados, señala mantener relaciones sexuales, no existiendo diferencias significativas entre chicos y chicas. El promedio de parejas de los que han mantenido relaciones sexuales es de 1.86. Es el preservativo el método anticonceptivo que utilizan mayoritariamente los que mantienen relaciones sexuales en la actualidad $(90.2 \%)$

Figura 4

\section{Método anticonceptivo utilizado por los que mantienen relaciones sexuales en la actualidad}

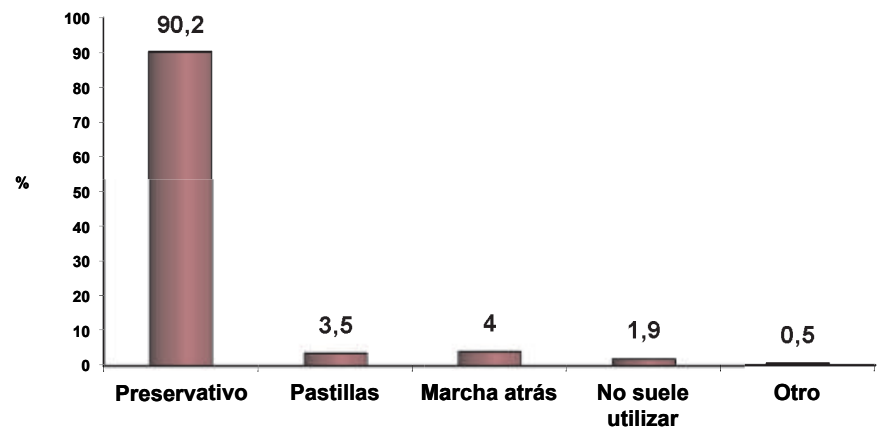

También consideramos de importancia mencionar que un 16.6\% señala haber hecho uso de métodos anticonceptivos, dirigidos únicamente a la prevención del embarazo no deseado, y tras haber mantenido la relación, como es el caso de la conocida "píldora del día después".

En la siguiente figura se puede observar el conocimiento de las enfermedades de transmisión sexual. Se puede concluir que el recibir educación sexual facilita la identificación de las distintas enfermedades de transmisión sexual, ya que aquellos alumnos que la recibieron, reconocen significativamente en mayor medida las diferentes ETS (Gonorrea, Herpes, Papiloma, Sífilis, VIH-SIDA, Clamidiasis, Tricomoniasis) que los que no recibieron dicha educación. 
Figura 5

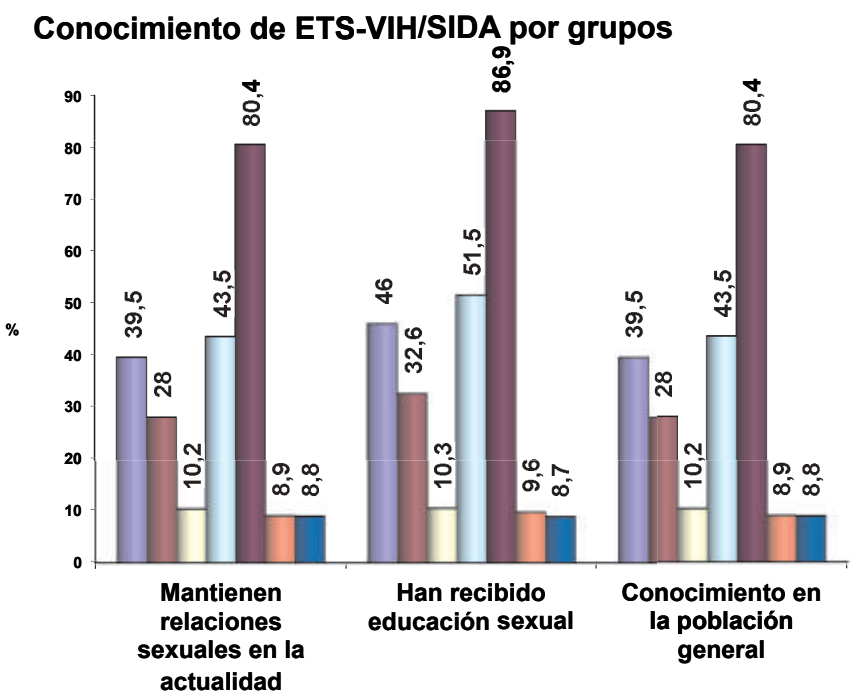

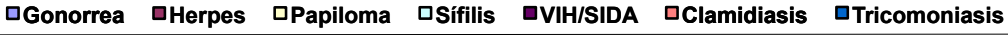

En el análisis de la incidencia que tiene la educación sexual en los alumnos estudiados, encontramos que si bien la educación sexual, como se ha visto, facilita un mayor conocimiento de las ETS, no genera en los alumnos la conciencia de usar medidas anticonceptivas que las eviten, como se ha expuesto con anterioridad.

Por último se analizó la existencia del conjunto de variables familiares, académicas y comunicativas, analizadas anteriormente, junto con la edad y sexo de manera que pudiésemos encontrar una influencia a modo de modelo de estas variables en relación al mantenimiento de relaciones sexuales completas.

Tabla $\mathrm{V}$

Modelo explicativo conducta sexual y variables familiares y académicas

Restumen del madeio

\begin{tabular}{|c|c|c|c|c|c|c|c|c|c|}
\hline \multirow[b]{2}{*}{ Modelo } & \multirow[b]{2}{*}{ R. } & \multirow[b]{2}{*}{ R. cuadrado } & \multirow[b]{2}{*}{$\begin{array}{c}\text { R. cuadrado } \\
\text { corregida }\end{array}$} & \multicolumn{6}{|c|}{ Estadistucos de cambso } \\
\hline & & & & $\begin{array}{l}\text { Error tip de } \\
\text { la estimación }\end{array}$ & $\begin{array}{c}\text { Cambio en } \mathrm{R} \\
\text { cuadrado }\end{array}$ & Cambio en $\mathrm{F}$ & g]2 & $\begin{array}{l}\text { Sig Cambio } \\
\text { en F }\end{array}$ & $\begin{array}{l}\text { Durbun- } \\
\text { Watson }\end{array}$ \\
\hline 1 & $411^{2}$ & .169 & .160 & .361 & .169 & 19,680 & 141356 & .000 & 2,073 \\
\hline \multicolumn{10}{|c|}{ 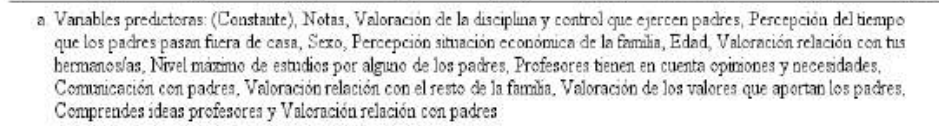 } \\
\hline b $\mathrm{Vec}$ & de & endiente: $\mathrm{Hs}$ & las tenido coits & & & & & & \\
\hline
\end{tabular}


El modelo que obtenemos en relación con esta conducta resulta ser significativo $F(14,1356)=19.68 ; p=0.00$ y con un buen nivel de varianza explicada $\left(R^{2}=0.169\right)$ de un $16.9 \%$. De entre las variables podemos destacar como más explicativas las siguientes, edad $(p=0.00)$, percepción de la situación económica de la familia $(\mathrm{p}=0.03)$, nivel máximo de estudios alcanzado por alguno de los padres 0 tutores $(\mathrm{p}=0.01)$, valoración de la relación con tus hermanos $(\mathrm{p}=0.01)$, percepción de que los profesores tienen en cuenta sus opiniones y necesidades $(p=0.00)$ y las notas $(p=0.00)$. Pertenecer a los primeros cursos de la ESO, tener una buena percepción de los profesores como personas que atienden necesidades y tienen en cuenta las opiniones, obtener buenas calificaciones, y un nivel mayor de estudios alcanzado por los progenitores se erigen como factores de protección de esta conducta, retrasando el inicio de la actividad sexual. Señalar que también los datos informan de que una mala percepción de la situación económica de la familia y una peor valoración de la relación con los hermanos, apuntan en el sentido de los factores de protección.

Tabla VI

Variables explicativas del modelo

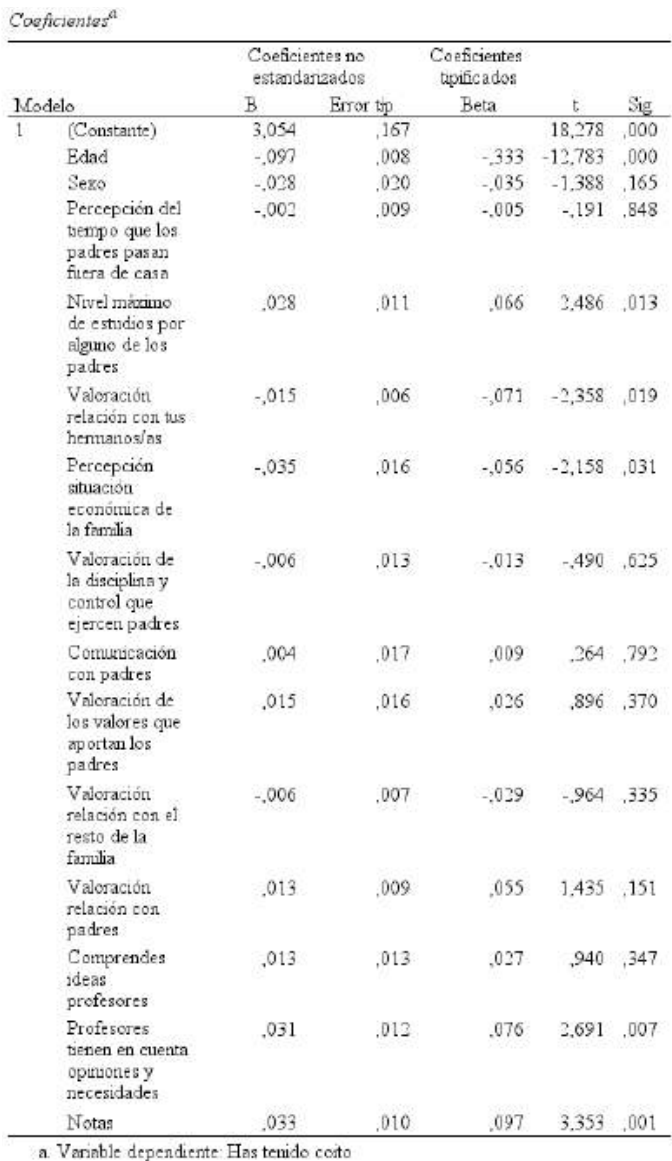




\section{DISCUSIÓN.}

Los datos referidos a la edad de inicio de la actividad sexual, que en nuestro trabajo se ubica en los 14.28 años (14.11 vs. 14.48), están en línea con los de la Encuesta Nacional de Salud Sexual (2009) que señala que en el tramo de 13-14 años un $13.2 \%$ dice haber tenido relación sexual y un $24.8 \%$ de los de entre 15 y 16 años. Por su parte, la encuesta Schering (2009) señala que entre el $15.8 \%$ de los chicos y el $15.7 \%$ de las chicas, que dicen haber tenido relaciones sexuales completas, se encuentra entre los 15 y 19 años.

Los padres se encuentran como la principal fuente de información sexual, seguido de los amigos. Estos datos van en una dirección distinta a la señalada por Sánchez y Muñoz (2005) quienes señalan a los amigos como fuente principal de información. Sin embargo, la encuesta Bayer Schering (2009) indica tanto a la familia, como a los amigos como fuente principal de información, tal y como se revela en nuestros datos.

Los jóvenes estudiantes que han mantenido relaciones completas valoran mejor la educación sexual que los que no. Además aquellos que manifiestan haber recibido educación sexual, valoran ésta de manera muy alta. Esto se encuentra en sintonía con los datos de la la 3 a Encuesta Bayer Shering (2009) en donde también señalan los encuestados una valoración de la información sexual buena 0 muy buena.

En nuestra muestra hemos señalado que el 17.4\% dice haber mantenido relaciones sexuales con penetración. En este sentido la encuesta HSCB (Moreno et al., 2011) señala que un $26.5 \%$ de chicos y chicas entre los 15-16 años y un 44.9\% de entre 17 y 18 años habrían realizado actividad sexual completa. A pesar de horquillas diferentes podemos señalar que la proporción en nuestro caso es algo menor.

Al igual que en nuestro estudio, la Encuesta Nacional de Salud Sexual (2009) señala que el 43\% la primera vez que lo hizo fue con su pareja y un $36 \%$ con alguien esporádico u ocasional.

El uso del preservativo se muestra similar al que nos apunta la Encuesta de Salud Sexual (2009) donde nos señala que un $32 \%$ utilizaron protección y el preservativo es el método mayoritario, seguido de la píldora y la marcha atrás. Similares datos apunta la encuesta Schering (2009) con un $62.3 \%$ de las chicas y un $33.7 \%$ de los chicos han utilizado algún método anticonceptivo siendo usado el preservativo por el $46.1 \%$ de las chicas y el $61.6 \%$ de los chicos. Señala también el estudio el uso de la píldora (7.7\%) y la "marcha atrás" (12.7\%).

Nuestros jóvenes que dicen haber recibido educación sexual no utilizan más el preservativo por ello, en su primera relación sexual. Además los que no han recibido educación sexual son más precoces en la realización de conductas sexuales de riesgo como la fellatio, no dándose el mismo resultado con otra práctica sexual de riesgo como el cunnilingus. Parece ser que la información sexual proveniente de fuentes como amigos, padres, medios de comunicación u otras fuentes presentan un importante papel, que en algunos casos es más decisivo que la información sexual recibida en los centros o de carácter institucional. Señalar también que aquellos que han recibido educación sexual reconocen mejor las distintas enfermedades de transmisión sexual, siendo la más conocida el VIH-SIDA.

En nuestro trabajo al igual que en la literatura, las variables del entorno familiar y escolar se presentan como factores de protección de la conducta sexual (White y Warner, 2015). En nuestro trabajo la buena valoración de los elementos familiares (padres y resto de la familia), así como la valoración de la comunicación y los valores transmitidos; y la disciplina y control que ejercen los padres, se convierten en un factor de protección de la conducta sexual temprana.

La familia, y su estructura, parece ejercer una influencia en la realización de conductas sexuales de riesgo en la adolescencia (Andrade, Betancourt y Palacios, 2006; Lameiras, Rodríguez, Calado y González, 2004; y Palacios y Andrade, 2006). 
En línea con nuestros resultados señalar el trabajo de (Malcolm et al., 2013) que pone de manifiesto que las buenas relaciones padres e hijos, cercanas y positivas retrasan la conducta sexual de riesgo, así como mejora el uso del preservativo; Cavazos-Rehg et al. (2010), señalan las relaciones disfuncionales entre padres e hijos, la supervisión paternal reducida y la falta de cohesión como elementos que potencian el riesgo de relaciones sexuales precoces. DiClemente et al. (2008) ponen el énfasis en la estructura familiar, el apoyo de la misma, su cohesión, la supervisión parental y la comunicación entre padres e hijos como factor de protección. Es importante hacer hincapié en la supervisión o monitoreo parental como mecanismo de control sobre los hijos que a su vez se presentará como factor de protección para la conducta sexual precoz (Dittus et al., 2015) .

En relación a las variables del entorno académico señalar que nuestros resultados participan, una vez más, de las aportaciones que hemos abordado en nuestro marco teórico. El trabajo de Manlove, Ryan y Franzetta (2007) indica que elementos del nivel educativo, como las bajas calificaciones y las expulsiones continuas son factores de riesgo para las conductas sexuales tempranas. Castro, Bermúdez, Buela-Casal y Madrid, (2011), Kotchick, Shaffer, Forehand y Miller, (2001), Sabia (2007), señalan que el apego y la buena adaptación al entorno escolar, y la buena integración de alumnos y profesores, unido a un buen rendimiento académico, constituyen un factor protector ante el inicio temprano de prácticas sexuales y los riesgos asociados al mismo.

Por último señalar, por un lado, que nuestros resultados muestran que hay una relación entre la mayor formación académica por parte de alguno de los padres y la no práctica de relaciones sexuales precoces. Ya Cavazos-Rehg et al. (2010) señalan que el nivel cultural y educativo de los padres se relaciona con el comportamiento sexual precoz de los hijos, encontrándose a mayor nivel formativo de los padres, un inicio más tardío de la sexualidad de éstos.

\section{CONCLUSIONES.}

Postergar el inicio de las relaciones sexuales en la adolescencia, en pos de un mayor desarrollo madurativo de la persona, supone un factor de protección de los riesgos asociados a dicha conducta. En relación con la educación sexual recibida, ésta se encuentra relacionada con un mejor reconocimiento de las distintas enfermedades de transmisión sexual. Además, los jóvenes que han mantenido relaciones sexuales completas manifiestan tener mejor percepción de la valoración de la educación sexual recibida que aquellos que no han mantenido relaciones completas. Sin embargo, el haber recibido educación sexual no es determinante para utilizar más, de manera significativa, un método anticonceptivo como el preservativo en su primera relación sexual.

En relación con la educación sexual como forma de prevención de los efectos no deseables del mantenimiento de conductas sexuales precoces, señalar que si bien parece efectiva para el reconocimiento de ETS, no parece que funcione de la misma manera para el uso del preservativo. En este caso es necesario aunar las distintas fuentes de información sexual para poder hacer hincapié, todas en el mismo sentido, en la necesidad de utilizar los métodos anticonceptivos en general, y el preservativo en particular, dada las circunstancias que rodean a los jóvenes andaluces en relación al mantenimiento de relaciones completas.

Por último, parece confirmarse que los entornos más cercanos al adolescente son vitales para protegerlo de conductas sexuales tempranas y de riesgo. Tanto la familia como el contexto escolar, así como las relaciones que se establecen entre sus actores fundamentales (padres, hermanos, profesores, compañeros, etc.) y las relaciones que se establecen en su seno con sus miembros a nivel de comunicación y monitoreo, como el papel de los docentes dentro del entorno escolar, no sólo a nivel de atención de los adolescentes, sino también como potenciador de una mejora del rendimiento académico del alumnado, se convierten en piezas claves a la hora de promover conductas sexuales sin riesgo en la adolescencia. 


\section{REFERENCIAS BIBLIOGRÁFICAS}

3를 encuesta Bayer Schering Pharma (2009). Sexualidad y anticoncepción en la juventud española. Equipo Daphne. https://goo.gl/NSdMuA

Andrade, P. P., Betancourt, 0. D., \& Palacios, D. J. (2006). Factores Familiares Asociados a la Conducta Sexual en Adolescentes. Revista Colombiana de Psicologia, 15, 91- 101. https://g00.gl/iZMC9R

Barkley, R .A., Fischer, M., Smallish, L., \& Fletcher, K. (2006). Young adult outcome of hyperactive children. Adaptive functioning in major life activities. Journal of the American Academy of Child and Adolescent Psychiatry. 45:192-202. http://dx.doi.org/10.1097/01. chi.0000189134.97436.e2

Cueto, S., \& Leon, J. (2016). Early sexual initiation among adolescents: A longitudinal analysis for 15-year-olds in Peru I La iniciación sexual precoz entre los adolescentes: Un análisis Iongitudinal de adolecentes de 15 años de edad en el Perú. Interamerican Journal of Psychology, 50(2). https://goo.gl/dNgCjk

Castro, A., Bermúdez, M. P., Buela Casal, G., \& Madrid, J. (2011). Variables psicosociales que median en el debut sexual de adolescentes en España. Revista Latinoamericana de Psicología, 43, (1), 83-94. https://goo.gl/Z3ZXii

Cavazos-Rehg, P. A., Spitznagel, E. L., Bucholz, K. K, Nurnberger, J., Edenbergf, H. J., Kramer, J. R, Kuperman, S., Hesselbrock, V., \& Bierut, L.J. (2010) Predictors of sexual debut at age 16 or younger. Archives of sexual behavior, 39(3):664-73. doi: 10.1007/s10508-008-9397-y

Crosby, R. A., DiClemente, R. J., Wingood, G. M., Lang, D. L., \& Harrington, K. (2003). Infrequent parental monitoring predicts sexually transmitted infections among low-income African American adolescent females. Archives of pediatrics \& adolescent medicine, 157(2), 169-173. doi: 10.1001/archpedi.157.2.169

Crosby, R. A., DiClemente, R. J., Wingood, G. M., Cobb, B. K., Harrington, K., Davies, S.L., Hook, E. W., \& Oh MK. (2002). Condom use and correlates of African American adolescent females' infrequent communication with sex partners about preventing sexually transmitted diseases and pregnancy. Health Education Behavioral, 29:219-31. Doi: 10.1177/109019810202900207

Cruces, S. J. (2012). Análisis integral de comportamientos de riesgo en población adolescente en Andalucía (Tesis Doctoral). Universidad de Cádiz.

DiClemente, R., Crittenden, C., Rose, E., Sales, J., Wingood, G. Crosby, R., \& Salazar, L. (2008). Psychosocial Predictors of HIV-Associated Sexual Behaviors and the Efficacy of Prevention Interventions in Adolescents at-Risk for HIV Infection: What Works and What Doesn't Work? Psychosomatic Medicine, 70:598-605. Doi: 10.1097/PSY.0b013e3181775edb

Dittus, P. J., Michael, S. L., Becasen, J. S., Gloppen, K. M., McCarthy, K., \& Guilamo-Ramos, V. (2015). Parental monitoring and its associations with adolescent sexual risk behavior: A metaanalysis. Pediatrics, 136(6). e1587-e1599 http://doi.org/10.1542/peds.2015-0305

Encuesta Nacional de Salud Sexual (2009). Ministerio de Sanidad, Servicios Sociales e Igualdad. https://goo.gl/rwAqfw

Erkut, S., Grossman, J. M., Frye, A. A., Ceder, I., Charmaraman, L., \& Tracy, A. J. (2013). Can Sex Education Delay Early Sexual Debut? Journal of Early Adolescence, 33(4) 482-497. http://doi.org/10.1177/0272431612449386

Espadas, J. P., Quiles, M. J., \& Méndez, F. J. (2003). Conductas sexuales de riesgo y prevención del sida en la adolescencia. Papeles del Psicólogo; 85. 29-36. https://goo.gl/aFAiuB

Espada, J. P., Escribano, S., Orgilés, M., Morales, A., \& Guillén-Riquelme, A. (2015). Sexual risk behaviors increasing among adolescents over time: Comparison of two cohorts in Spain. AIDS 
Care - Psychological and Socio-Medical Aspects of AIDS/HIV, 27(6)783-788. http://dx.doi.org/10.1080/09540121.2014.996516

Ferreira, M., Nelas, P., Duarte, J., Albuquerque, C., Grilo, C., \& Nave, F. (2013). Family culture and adolescent sexuality. Atencion Primaria, 45(SUPPL. 2) 216-222. https://goo.gl/ZRRHXU

Moreno, C., Ramos, P., Rivera, F., Muñoz-Tinoco, V., Sánchez-Queija, I., Granado, M. C., \& JiménezIglesias, A. (2011). Desarrollo adolescente y salud en España. Resumen del estudio Health Behaviour in School-aged Children (HBSC - 2006). Madrid, España: Ministerio de Sanidad, Política Social e Igualdad. NIP0: 860110366. https://goo.gl/kyffbe

Instituto de Salud Carlos III. (2011). Vigilancia epidemiológica del VIH/SIDA en España. Ministerio de Sanidad, Política Social e Igualdad. Actualización 30 de Junio de 2011. http://www.msps.es/novedades/docs/InformeVIH-sida Junio2011.pdf

Kotchick, B. A., Shaffer, A. N., Forehand, R., \& Miller, K. S. (2001). Adolescent sexual risk behavior: A multi-system perspective. Clinical Psychology Review. 21:493-519. http://dx.doi.org/10.1016/S0272-7358(99)00070-7

Lameiras, M., Rodríguez, Y., Calado, M., \& González, M. (2004) Determinantes del inicio de las relaciones sexuales en adolescentes españoles. Cuadernos de Medicina Psicosomática y Psiquiatría de Enlace, 68,71-72.

Lindberg, L. D., Maddow-Zimet, I., \& Boonstra, H. (2016). Changes in Adolescents' Receipt of Sex Education, 2006-2013. Journal of Adolescent Health, 58(6), 621-627. http://doi.org/10.1016/j.jadohealth.2016.02.004

Malcolm, S., Huang, S., Cordova, D., Freitas, D., Arzon, M., Jimenez, G. L., ... Prado, G. (2013). Predicting Condom Use Attitudes, Norms, and Control Beliefs in Hispanic Problem Behavior Youth: The Effects of Family Functioning and Parent-Adolescent Communication About Sex on Condom Use. Health Education and Behavior, 40(4): 384-391. http://doi.org/10.1177/1090198112440010

Manlove, J.S., Ryan, S., \& Franzetta, K. (2007). Risk and protective factors associated with the transition to a first sexual relationships with an older partner. Journal of Adolescent Health, 40, 135143. Doi: 10.1016/j.jadohealth.2006.09.003

Newman, K., Harrison, L., Dashiff, C., \& Davies S. (2008) Relationships between parenting styles and risk behaviors in adolescent health: an integrative literature review. Rev Latino-am Enfermagem. Enero-Febrero, 16(1),142-50. http://dx.doi.org/10.1590/S010411692008000100022

Palacios, D. J., \& Andrade, P. P. (2006). Diferencias en los Estilos parentales y la conducta sexual de riesgo en adolescentes. En: Sánchez, A. R., Díaz-Loving, R. y Rivera, A. S. (Eds.). La Psicologia Social en México, 11, 775- 781. México: AMEPSO.

Ramrakha, S., Bell, M. L., Paul, C., Dickson, N., Moffitt, T .E., \& Caspi, M. (2007). Childhood behavior problems linked to sexual risk taking in young adulthood: A birth cohort study. Journal of the American Academy of Child and Adolescent Psychiatry. 46,1272-1279. Doi: 10.1097/chi.0b013e3180f6340e

Rodriguez, J, \& Traverso, C. I. (2012). Conductas sexuales en adolescentes de 12 a 17 años de Andalucía. Gaceta Sanitaria; 26,519-24. http://dx.doi.org/10.1016/i.gaceta.2012.02.005

Rodríguez, J., Bárcena, G., Cruces, S.J., Hernández, P., Jiménez, I., Tinoco, J., \& Traverso. C. (2008). Comportamientos de Riesgo (violencia, consumo de alcohol y drogas y conductas sexuales) en estudiantes de ESO de Andalucía. Resultados Preliminares. Málaga: Grupo 33. https://g00.gl//yVGgJl

Sabia, J. J. (2007). Early adolescent sex and diminished school attachment: Selection or spillovers? 
Southern Economic Journal, 74, 239-268. Doi: 10.2307/20111962

Salazar, I. C., Varela, M. T., Tovar, J. R. \&, Cáceres, D. E. (2006). Construcción y validación de un cuestionario de factores de riesgo y de protección para el consumo de drogas en jóvenes universitarios. Acta Colombiana de Psicología, 9, 19-30. https://g00.gl/ttXg71

Sanchez, M., \& Muñoz, A. (2005). Influencia de padres y amigos sobre la actitud hacia las conductas sexuales de prevención en la adolescencia. Un análisis en función del género. Revista Latinoamericana de Psicología, 37, (1), 71-79. https://goo.gl/LLZTQZ

Silver, E. J., \& Bauman, L. J. (2006) The association of sexual experience with attitudes, beliefs, and risk behaviors of inner-city adolescents. Journal of Research on Adolescence, 16(1), 29-45. Doi: 10.1111/j.1532-7795.2006.00118.x

Temple-Smith, M., Moore, S., \& Rosenthal, D. (2015). Sexuality in Adolescence: The Digital Generation. Sexuality in Adolescence: The digital generation. http://doi.org/ $10.4324 / 9781315849348$

Teva, I.; Bermúdez, M., \& Buela-Casal, G. (2009). Variables sociodemográficas y conductas de riesgo en la infección por el VIH y las enfermedades de transmisión sexual en adolescentes: España, 2007. Revista. Española de Salud Publica83 (2), 309-320. https://goo.gl/KOAcfW

UNAIDS (2016). Informe de brechas en Prevención. https://goo.gl/WHPf9K

White, C. N., \& Warner, L. A. (2015). Influence of family and school-level factors on age of sexual initiation. Journal of Adolescent Health, 56(2), 231-237.. http://doi.org/10.1016/j.jadohealth.2014.09.017 